\title{
Index to Volume 120
}

\section{Compiled by Leslie Cody}

Abies amabilis, 172

balsamea, 100,236,238,299,433,440

lasiocarpa, 100,331

Accipiter striatus, 46

Acer spp., 226

circinatum, 11,171,348

glabrum var. douglasii, 171

macrophyllum, 11,180

rubrum, 299,354

saccharum, 299

Achillea millefolium, 354

Achnatherum hymenoides, 336

Acipenser fulvescens, 71

$$
\text { transmontanus, } 422
$$

Acipenser fulvescens, Movements in Rainy Lake, Minnesota and Ontario, Lake Sturgeon, 71

Acorn, J., Reviews by, 245,489

Adams, W.E. Jr., L.W. Kallemeyn, and D.W. Willis. Lake Sturgeon, Acipenser fulvescens, Movements in Rainy Lake, Minnesota and Ontario, 71

Adiantum capillus-veneris, 138

Aegolius acadicus, 294,303

Aeshna sp., 416

canadensis, 415

constricta, 417

eremita, 417

interrupta, 416

interrupta lineata, 416

interrupta lineata ssp. lineata, 416

umbrosa, 416

umbrosa umbrosa, 417

Agalinis neoscotica, 357

Agriotes collaris, 19 stabilis, 19

Agropyron cristatum, 337

Agrostis exerata, 214 tenuis, 354

Agujaceratops, 403

Aira caryophyllea, 320 praecox, 154,320

Aix sponsa, 236,303

Alaska, First Record of a River Otter, Lontra canadensis, Captured on the Northeastern Coast of, 235

Alaska, Physical Characteristics, Hematology, and Serum Chemistry of Free-ranging Gray Wolves, Canis lupus, in Southcentral, 205

Alaska, 2001-2003, Assessment of Effects of an Oil Pipeline on Caribou, Rangifer tarandus granti, Use of Riparian Habitats in Arctic, 323

Alberta, A New Record of Deepwater Sculpin, Myoxocephalus thompsonii, in Northeastern, 480

Alberta Boreal Forests, Winter Habitat Use by American Marten, Martes americana, in Western, 100

Alberta, Pack Size of Wolves, Canis lupus, on Caribou, Rangifer tarandus, Winter Ranges in Westcentral, 313
Alces alces, 205,313,367

alces americana, 51

Alder, 236,240

Red, 11,348

Speckled, 22

Allium amplectens, 160

cernuum, 154

Alnus sp., 236,238,240

incana, 22

rubra, 348

rubus, 11

Alopecurus carolinianus, 164

Alopex lagopus, 36

Alosa sapidissima, 422

Ambystoma gracile, 90 macrodactylum, 90

Ameiurus catus, 424

melas, 422

natalis, 421

nebulosus, 422

Ameiurus natalis, a Loricariid Catfish, Panaque suttonorum, and a Silver Pacu, Piaractus cf. P. brachypomus, in British Columbia, First Records of the Yellow Bullhead, 421

Amelanchier, 428

alnifolia, 176,428

alnifolia var. compacta, 428

florida, 428

humilis, 428

humilis var. compacta, 428

laevis, 354

laevis $\times$ lucida, 354

lucida, 354

sanguinea, 428

Amelanchier in the Whitewood area of Southeastern Saskatchewan and the First Saskatchewan Records of Amelanchier sanguinea, Regional Variation in, 428

Amelanchier sanguinea, Regional Variation in Amelanchier in the Whitewood area of Southeastern Saskatchewan and the First Saskatchewan Records of, 428

Amia calva, 422

Ammodytes americanus, 96

Ammospiza leconteii, 22

Ammospiza leconteii, from Northeastern Ontario, with Some Notes on Nesting Behaviour, New Nesting Records of the Le Conte's Sparrow, 22

Anaphalis margaritacea, 354

Anas clypeata, 5

platyrhynchos, 6

Anax junius, 417

Anderson, C.S., D.B. Meikle, A.B. Cady, and R.L. Schaefer Annual Variation in Habitat Use by White-footed Mice, Peromyscus leucopus: The Effects of Forest Patch Size, Edge and Surrounding Vegetation Type, 192

Andrena, 19 
Anguilla rostrata, 238,477

Anguille d'Amérique, 238

Antennaria flagellaris, 183 howellii ssp. neodioica, 354

Antennaria flagellaris, in Canada, Conservation Evaluation of Stoloniferous Pussytoes, 183

Anthophora, 180

Anthoxanthum odoratum, 148,154,160

Antilocapra americana, 210

Apera interrupta, 320

Apis mellifera, 294

Apocynum androsaemifolium var. incanum, 354

Apodemus sylvaticus, 195

Apple-Producing Region of Southern Quebec, First Observations of an Eastern Screech-Owl, Megascops asio, Population in an, 289

Aralia hispida, 354

Arbutus menziesii, 148,180

Arbutus, 148,180

Arctostaphylos uva-ursi, 172,336,354

Arenaria capillaris ssp. americana, 184

Aronia sp., 353

Aronia, 360

Artemisia cana ssp. cana, 336 tridentata, 164,184

Ascophyllum nodosum, 236

Asio otus, 295

Aspen, 314,428 Trembling, 22,100,342

Astragalus miser, 176

Athous acanthus, 19

Auditor's Report, 398

Aulnes, 238

Aythya sp., 6

affinis, 5

americana, 109

collaris, 6

marila, 109

valisneria, 109

Baccha elongata, 17

Badger, 209

American, 31,342

European, 52

Badiou, P.H.J. and L.G. Goldsborough. Northern Range Expansion and Invasion by the Common Carp, Cyprinus carpio, of the Churchill River System in Manitoba, 83

Balaena mysticetus, 462

Balaenoptera acutorostrata, 95

borealis, 97

musculus, 95

physalus, 95

Balanus improvisus, 106

Baldwin, R.A., A.E. Houston, M.L. Kennedy, and P.S. Liu. Predicting Raccoon, Procyon lotor, Occurrence Through the Use of Microhabitat Variables, 225

Balkwill, D., B.W. Coad, I. Galvez, and J. Gilhen. First Record of the Great Barracuda, Sphyraena barracuda from Canada, 241

Ballard, W.B., 205

Balsamorhiza sagittata, 176

Balsamroot, Arrow-leaved, 176

Barley, 343

Barracuda, Great, 241

Barracuda, Sphyraena barracuda from Canada, First Record of the Great, 241
Barton, K., 163,183

Bartonia virginica, 357

Basquill, S., 351

Bass, Largemouth, 422

Smallmouth, 421

Striped, 422

Bat, Big Brown, 39

Little Brown, 39,51

Long-legged, 39

Northern Long-eared, 39,51,332

Silver-haired, 39

Western Long-eared, 39

Bat, Myotis septentrionalis, in the Yukon Territory, First Records of the Northern Long-eared, 39

Bdellarogatis, 444 plumbeus, 444

Bear, American Black, 51 Black, 57,68,110,209,314

Brown, 57

Grizzly, 314

Bear, Ursus americana, Retrieval of an Elk, Cervus elaphus, Carcass from a Small Lake in Riding Mountain National Park, Manitoba, Probable Black, 110

Bear, Ursus americanus L., Long-range Homing by an Adult Female Black, 57

Bearberry, Common, 336

Beaudoin, A.B., Reviews by, 383,493,494

Beaver, 51,62,205,331,368

Bee, 15

Honey, 294

Beetle, 296

Click, 15

Ground, 16

Beluga, 457

Bertrand, A.-S., S. Kenn, D. Gallant, E. Tremblay, L. Vasseur, and R. Wissink. MtDNA Analyses on Hair Samples Confirm Cougar, Puma concolor, Presence in Southern New Brunswick, Eastern Canada, 438

Betula spp., 428,440

alleghaniensis, 299

cordifolia, 299

papyrifera, 22,50,238,299,354,433,440

populifolia, 354

Birch, 428,440

Mountain, 299

Paper, 433

White, 22,50,299,440

Yellow, 299

Bird, D.M., 289

Bison bison athabascae, 68

Bison, Wood, 68

Bitter-cress, Little Western, 180

Bizecki Robson, D. A Conservation Evaluation of Smooth Goosefoot, Chenopodium subglabrum (Chenopodiaceae), in Canada, 335

Blackberry, 359

Blaney, S., 319

Blarina brevicauda, 294,436

Blastocerms dichotomus, 64

Blinn, B.M., V. Violette, and A.W. Diamond. Osprey, Pandion haliaetus, Depredates Common Eider, Somateria mollissima, Duckling, 236

Blue-eyed Mary, Large-flowered, 180

Blue-Eyed-Grass, Coastal-Plain, 359

Blueberry, 360

Alaskan, 172 
Bluegill, 422

Bluegrass, Annual, 320

Bulbous, 320

Kentucky, 160

Bluejoint, Canada, 22

Bluet, Azure, 417

Big, 417

Familiar, 417

Hagen's, 417

Marsh, 417

Blush, Sea, 154,158

Bobcat, 230

Bobwhite, Northern, 452

Bobwhites, Colinus virginianus, by Mammalian Mesopredators: Does the Problem-Individual Paradigm Fit?, Predation on Artificial Nests of Northern, 452

Bombus, 180 perplexus, 19

vagans, 19

Bombycilla cedrorum, 294

Boreal Dip Net/L'Epuisette Borèale January 2006, The, 392

Bouleau blanc, 238

Bowfin, 422

Brachyopa, 20 notata, 17

Brachypalpus oarus, 17

Branta canadensis, 109

British Columbia, A Test of Interspecific Effects of Introduced Eastern Grey Squirrels, Sciurus carolinensis, on Douglas's Squirrels, Tamiasciurus douglasii, in Vancouver, 10

British Columbia, Early Ontogenetic Diet in Gray Wolves, Canis lupus, of Coastal, 61

British Columbia, First Records of the Yellow Bullhead, Ameiurus natalis, a Loricariid Catfish, Panaque suttonorum, and a Silver Pacu, Piaractus cf. P. brachypomus, in, 421

British Columbia, Life History Phenology and Sediment Size Association of the Dragonfly Cordulegaster dorsalis (Odonata: Cordulegastridae) in an Ephemeral Habitat in Southwestern, 347

Brome, 148,154

Barren, 160,180

Bromus, 148,154 sterilis, 160,180

tectorum, 176

Broom, Scotch, 148,155,160

Brunelle, P.M., 413

Bryan, H.M., C.T. Darimont, T.E. Reimchen, and P.C. Paquet. Early Ontogenetic Diet in Gray Wolves, Canis lupus, of Coastal British Columbia, 61

Bryant, J.E. A Tribute to Nicholas Stephen Novakowski 1925-2004, 370

Bubo virginianus, 290

Bucephala albeola, 6 clangula, 5,109

Buckwheat, 343

Cushion, 184

Parsnip-flowered, 184

Budworm, Spruce, 50,299

Bufflehead, 6

Bufo americanus, 240 boreas, 87

Bullhead, Black, 422

Brown, 422
Yellow, 421

Bullhead, Ameiurus natalis, a Loricariid Catfish, Panaque suttonorum, and a Silver Pacu, Piaractus cf. P. brachypomus, in British Columbia, First Records of the Yellow, 421

Bulrush, American Three-square, 213

Bunchberry, 440

Bunker-Popma, K. Scoter, Melanitta spp., Migrations Interrupted by Confederation Bridge: An Update, 232

Bur-reed, Narrowleaf, 240

Burbot, 422,480

Burchsted, F., 106

Burchsted, J.C.A. and F. Burchsted. Lady Crabs, Ovalipes ocellatus, in the Gulf of Maine, 106

Butcher, M.K., 323

Butler, M.J., W.B. Ballard, and H.A. Whitlaw. Physical Characteristics, Hematology, and Serum Chemistry of Free-ranging Gray Wolves, Canis lupus, in Southcentral Alaska, 205

Buttercup, Bur, 319

Buttercup, Ranunculus testiculatus, New to Eastern Canada, Bur, 319

Butterfly, Maritime Copper, 419

Cabbage, Skunk, 172

Cady, A.B., 192

Calamagrostis sp., 354 canadensis, 22

Calamovilfa longifolia, 336

Calidris spp., 5

Caligus, 199 clemensi, 199

Camarasaurus, 408

Camas, Common, 148

Camassia quamash, 148

Canada, A Conservation Evaluation of Smooth Goosefoot, Chenopodium subglabrum (Chenopodiaceae), in, 335

Canada, Bur Buttercup, Ranunculus testiculatus, New to Eastern, 319

Canada, Conservation Evaluation of Pacific Rhododendron, Rhododendron macrophyllum, in, 169

Canada, Conservation Evaluation of Slender Collomia, Collomia tenella, in, 175

Canada, Conservation Evaluation of Stoloniferous Pussytoes, Antennaria flagellaris, in, 183

Canada, Conservation Evaluation of the Bog Bird's-foot Trefoil, Lotus pinnatus, in, 157

Canada, Conservation Evaluation of the Pacific Population of Dwarf Woolly-heads, Psilocarphus brevissimus var. brevissimus, in, 163

Canada, Conservation Evaluation of the Prairie Lupine, Lupinus lepidus var. lepidus, in, 147

Canada, Conservation Evaluation of the Seaside Birds-foot Trefoil, Lotus formosissimus, in, 153

Canada, Conservation Evaluation of the Small-flowered Tonella, Tonella tenella, in, 179

Canada, Extension of Coyote, Canis latrans, Breeding Range in the Northwest Territories, 67

Canada, First Record of the Great Barracuda, Sphyraena barracuda from, 241

Canada, MtDNA Analyses on Hair Samples Confirm Cougar, Puma concolor, Presence in Southern New Brunswick, 438

Canada's Maritime Provinces, Recent Declines of House Sparrows, Passer domesticus, in, 43 
Canadian Arctic, Reactions of Narwhals, Monodon monoceros, to Killer Whale, Orcinus orca, Attacks in the Eastern, 457

Canadian Association of Herpetologists/Association Canadienne des Herpetologistes Bulletin, 502

Canadian Field-Naturalist, Advice for Contributors to The, $134,262,402,534$

Canadian Field-Naturalist 119(3), Errata The, 133

Canadian Field-Naturalist 120(1), Errata The, 259

Canadian Field-Naturalist 120(2), Errata The, 503

Canadian Field-Naturalist 120(3), Errata The, 503

Cancer magister, 364 productus, 363

Canis sp., 263 familiaris, 263479 latrans, 27,31,40,50,67,110,209,263,314,342,472,474, 478

lupus, 27,57,61,67,111,205,263,313,367,466,471,476, 478

lupus arctos, 466,47

lupus beothucus, 50

lycaon, 27

rufus, 27

Canis latrans, An Ethogram Developed on Captive Eastern Coyotes, 263

Canis latrans, Breeding Range in the Northwest Territories, Canada, Extension of Coyote, 67

Canis latrans, Conditions for Sexual Interactions Between Wild Grey Wolves, Canis lupus, and Coyotes, 27

Canis latrans, in a Heavily Urbanized Area: Implications for Ecosystem Management, Use of "Micro"-Corridors by Eastern Coyotes, 474

Canis latrans, in an Urban Area, Rat Poison Kills a Pack of Eastern Coyotes, 478

Canis lupus, and Coyotes, Canis latrans, Conditions for Sexual Interactions Between Wild Grey Wolves, 27

Canis lupus arctos: Influence of Order on Posture, Double Marking in Arctic Wolves, 471

Canis lupus arctos, in Summer, Urine-marking and Ground-scratching by Free-ranging Arctic Wolves, 466

Canis lupus, Group for Ontario, A New Record Size Wolf, 367

Canis lupus, in Southcentral Alaska, Physical Characteristics, Hematology, and Serum Chemistry of Free-ranging Gray Wolves, 205

Canis lupus, of Coastal British Columbia, Early Ontogenetic Diet in Gray Wolves, 61

Canis lupus, on Caribou, Rangifer tarandus, Winter Ranges in Westcentral Alberta, Pack Size of Wolves, 313

Canola, 343

Canvasback, 109

Carassius auratus, 422

Carbyn, L., Review by, 249

Carbyn, S., 307

Carbyn, S., P.M. Catling, S.P. Vander Kloet, and S. Basquill. An Analysis of the Vascular Flora of Annapolis Heathlands, Nova Scotia, 351

Cardamine oligosperma, 180

Cardinal, Northern, 294

Cardinalis cardinalis, 294

Carduellis tristis, 294

Carex atlantica, 357

cumulata, 354

deflexa var. deflexa, 354

emonsii, 354 feta, 160

lucorum, 360

lyngbyei, 213

nigra, 354

oligosperma, 22

paleacea, 416

praegracilis, 310

scoparia, 354

siccata, 360

stipata, 22

tonsa, 358

tonsa var. rugosperma, 354

tonsa var. tonsa, 354

tumulicola, 160

Caribou, 313,323

Barren-ground, 205

Woodland, 50,313,367

Caribou, Rangifer tarandus granti, Use of Riparian Habitats in Arctic Alaska, 2001-2003, Assessment of Effects of an Oil Pipeline on, 323

Caribou, Rangifer tarandus, Winter Ranges in Westcentral Alberta, Pack Size of Wolves, Canis lupus, on, 313

Carp, Common, 83,422

Carp, Cyprinus carpio, of the Churchill River System in Manitoba, Northern Range Expansion and Invasion by the Common, 83

Carver, D.B., 452

Carya spp., 226

Castor canadensis, 62,205,331,368

canadensis caecator, 51

Cat, Domestic, 46

Cat's-ear, Hairy, 154

Caterpillar, Forest Tent, 433

Catfish, Black, 425

Blue, 424

Brown, 425

Channel, 422

Flathead, 424

Loricariid, 421

White, 424

Yellow, 425

Catfish, Panaque suttonorum, and a Silver Pacu, Piaractus cf. P. brachypomus, in British Columbia, First Records of the Yellow Bullhead, Ameiurus natalis, a Loricariid, 421

Catling, P., Review by, 249

Catling, P.M., 351

Catling, P.M. and G. Mitrow. Regional Variation in Amelanchi$e r$ in the Whitewood area of Southeastern Saskatchewan and the First Saskatchewan Records of Amelanchier sanguinea, 428

Catling, P.M. and S. Carbyn. Recent Invasion, Current Status and Invasion Pathway of European Common Reed, Phragmites australis subspecies australis, in the Southern Ottawa District, 307

Catling, P.M., R. Hutchinson, and P.M. Brunelle. Use of Saltmarsh by Dragonflies (Odonata) in the Baie des Chaleurs Region of Quebec and New Brunswick in Late Summer and Autumn, 413

Catostomus catostomus, 422,481 commersoni, 422

Cedar, White, 300

Celithemis elisa, 417

Centaurea nigra, 354

Centrosaurus apertus, 408

Cerastium pumilum, 320 
semidecandrum, 320

Ceratocephalus testiculatus, 319

Ceratodon purpureus, 358

Certhia americana, 303

Cervus elaphus, 110,188,314

Cervus elaphus, Calf Observed Near Bancroft, Ontario, Lateborn Elk, 188

Cervus elaphus, Carcass from a Small Lake in Riding Mountain National Park, Manitoba, Probable Black Bear, Ursus americana, Retrieval of an Elk, 110

Chalcosyrphus, 20 nemorum, 17

Chamaecyparis nootkatensis, 172

Chamaedaphne calyculata, 23

Channa sp., 424

Char [Charr], Arctic, 199,235,422

Chasmosaurus, 403

$$
\text { belli, } 403
$$

humeri, 408

irvinensis, 403

mariscalensis, 403

russelli, 403

Chasmosaurus belli (Dinosauria: Ornithischia), A Reevaluation of Sexual Dimorphism in the Postcranium of the Chasmosaurine Ceratopsid, 403

Cheatgrass, 176

Cheilosia rita, 17 tristis, 17

Chen caerulescens caerulescens, 213

Chen caerulescens caerulescens, Exceeding the Carrying Capacity of the Fraser River Delta's Brackish Marshes?, Are Lesser Snow Geese, 213

Chenopodium album, 337

bonus-henericus, 340

fremontii, 340

pratericola, 336

quinoa, 340

subglabrum, 335

Chenopodium subglabrum (Chenopodiaceae), in Canada, A Conservation Evaluation of Smooth Goosefoot, 335

Cherry, 360 Pin, 50

Chickadee, Black-capped, 294,302 Boreal, 302

Chickweed, Curtis' Mouse-ear, 320 Lesser, 320 Small Mouse-ear, 320

Chimpanzee, 264

Chipmunk, 264,295 Eastern, 51

Chitonotus pugetensis, 364

Choristoneura fumiferana, 50,299

Chub, Flathead, 422

$$
\text { Lake, } 422
$$

Cifuni, S.M., 478

Circus cyaneus, 109

Cisco, 480 Shortjaw, 482

Cladina rangiferina, 358 stellaris, 358

Cladonia spp, 171

Cladonia, 171

Clarias sp., 424

Clarke, J.B., Review by, 247

Classen, M.-C., 347

Claytonia perfoliata, 180
Cleavers, 180

Clethrionomys sp., 291 gapperi, 50,331

Clethrionomys gapperi, in Newfoundland: Implications for the Endangered Newfoundland Marten, Martes americana atrata, First Record of the Southern Red-Backed Vole, 50

Clintonia borealis, 357

Cluff, H.D. Extension of Coyote, Canis latrans, Breeding Range in the Northwest Territories, Canada, 67

Clupea harengus, 96

Coad, B.W., 241

Coad, B.W. and A.M. Murray, Review by, 116

Coad, B.W., Review by, 114

Cody Receives Yukon Biodiversity Awareness Award, Bill, 258

Coenonypha tullia nipisiquit, 419

Colaptes auratus, 294,303

Coleoptera, 296

Colinus virginianus, 452

Colinus virginianus, by Mammalian Mesopredators: Does the Problem-Individual Paradigm Fit?, Predation on Artificial Nests of Northern Bobwhites, 452

Collinsia, 180 grandiflora, 180

Collomia linearis, 176 tenella, 175

Collomia, Narrow-leaved, 176 Slender, 175

Collomia, Collomia tenella, in Canada, Conservation Evaluation of Slender, 175

Collomia tenella, in Canada, Conservation Evaluation of Slender Collomia, 175

Colorado, Multiple Scale Den Site Selection by Swift Foxes, Vulpes velox, in Southeastern, 31

Colossoma sp., 424 bidens, 424

Columba spp., 57 livia, 294

Comptonia peregrina, 354

Coot, 2

Cordulegaster bidentata, 350 boltonii, 347 dorsalis, 347 heros, 350 maculata, 347

Cordulegaster dorsalis (Odonata: Cordulegastridae) in an Ephemeral Habitat in Southwestern British Columbia, Life History Phenology and Sediment Size Association of the Dragonfly, 347

Coregonus artedi, 480 clupeaformis, 422,480 zenithicus, 482

Corema, 357 conradii, 351

Corn, 226,294

Cornus canadensis, 354,440

Corvus brachyrhyncos, 109 corax, 109,110

Corylus cornuta, 170

Cotton, 226

Cottontail, Nuttall's, 165

Cottonwood, 226

Cottus asper, 425

Couesius plumbeus, 422

Cougar, 40,314,438,476

Eastern, 438 
Cougar, Puma concolor, Presence in Southern New Brunswick, Eastern Canada, MtDNA Analyses on Hair Samples Confirm, 438

Cow-wheat, 170

Cowbird, Brown-headed, 294

Coyote, 27,31,40,67,110,209,314,342,472 Eastern, 50,263,474,478

Coyote, Canis latrans, Breeding Range in the Northwest Territories, Canada, Extension of, 67

Coyotes Canis latrans, An Ethogram Developed on Captive Eastern, 263

Coyotes, Canis latrans, Conditions for Sexual Interactions Between Wild Grey Wolves, Canis lupus, and, 27

Coyotes, Canis latrans, in a Heavily Urbanized Area: Implications for Ecosystem Management, Use of "Micro"Corridors by Eastern, 474

Coyotes, Canis latrans, in an Urban Area, Rat Poison Kills a Pack of Eastern, 478

Crab, Dungeness, 364 Lady, 106

Redrock, 363

Crabs, Ovalipes ocellatus, in the Gulf of Maine, Lady, 106

Crane, Sandhill, 68

Crappie, Black, 422

Crataegus cf. chrysocarpa, 354

Creeper, Brown, 302

Crepis tectorum, 354

Crins, W.J., 15

Crocuta croenta, 52

Cronin, M.A., 323

Crow, American, 109

Ctenicera cruciatus, 20 hieroglyphica, 19 insidiosa, 19 resplendens, 19 spinosa, 19 triundulata, 18 vulnerata, 18

Cudweed, Lowland, 164

Culaea inconstans, 422

Curran, W.J., 50

Cutthroat, West-slope, 421

Cyanocitta cristata, 294

Cyclopterus lumpus, 464

Cygnus buccinator, 220

Cynomys ludovicianus, 31

Cynosurus echinatus, 148

Cyprinella lutrensis, 424

Cyprinus carpio, 83,422

Cyprinus carpio, of the Churchill River System in Manitoba, Northern Range Expansion and Invasion by the Common Carp, 83

Cypripedium acaule, 354

Cytisus scoparius, 148,155,160

Dactylis glomerata, 154,160

Daisy, Cut-leaved, 184 Salish, 137

Dalopius spp., 19 cognatus, 19 fuscipes, 19

Dandelion, Common, 320

Danthonia spicata, 354 unispicata, 164,184

Darimont, C.T., 61

Darner, Canada, 417
Common Green, 417

Lake, 417

Lance-tipped, 417

Shadow, 417

Variable, 417

Dasysyrphus pauxillus, 17

Davies, S., A. Griffiths, and T.E. Reimchen. Pacific Hagfish, Eptatretus stoutii, Spotted Ratfish, Hydrolagus colliei, and Scavenger Activity on Tethered Carrion in Subtidal Benthic Communities off Western Vancouver Island, 363

Deer, 68

Black-tailed, 63

Marsh, 64

Mule, 40,314

Pampas, 64

Sitka Black-tailed, 61

White-tailed, 29,40,64,68,209,313,367

Delphinapterus leucas, 457

Delphinium menziesii, 154

Delphinus delphis, 97

Demarchi, M.W. Are Lesser Snow Geese, Chen caerulescens caerulescens, Exceeding the Carrying Capacity of the Fraser River Delta's Brackish Marshes?, 213

Deschampsia danthonioides, 164 flexuosa, 354

Desert-parsley, Swale, 184

Desroches, J.-F. Extension de l'aire de distribution de l'Umbre de vase, Umbra limi, dans le nord-est du Québec, 238

Desroches, J.-F., I. Picard, and J.E. Maunder. The Mink Frog, Rana septentrionalis, in Southeastern Labrador, 239

Desrosiers, J., 169

Dewey, T.A., 39

Diamond, A.W., 236

Dianthus armeria, 354

Dichanthelium (sub Panicum) depauperatum var. psilophyllum $\mathrm{f}$. cryptostachys, 360

acuminatum var. fasciculatum, 354

depauperatum, 354

linearifolium, 354

sabulorum var. thinium, 360

Dicranum sp., 180

scoparium, 358

Dicrostonyx groenlandicus, 466

Didelphis virginiana, 229,452

Diervella lonicera, 354

Distichlis spicata, 214

Doellingeria umbellata var. umbellata, 354

Dog, 479

African Wild, 52

Domestic, 263

Dogtail, Hedgehog, 148

Dollar, Silver, 424

Dolphin, Atlantic White-sided, 95

Common Bottlenose, 97

Dusky, 97

Short-beaked Common, 97

White-beaked, 95

White-sided, 95

Donaldson, M.R., Review by, 121

Donovan, M. Conservation Evaluation of the Bog Bird's-foot Trefoil, Lotus pinnatus, in Canada, 157

Dorocordulia lepida, 417

Douglas, G.W. and J. Desrosiers. Conservation Evaluation of Pacific Rhododendron, Rhododendron macrophyllum, in Canada, 169 
Douglas, G.W. and J.L. Penny. Conservation Evaluation of Slender Collomia, Collomia tenella, in Canada, 175

Douglas, G.W. and J.L. Penny. Conservation Evaluation of the Small-flowered Tonella, Tonella tenella, in Canada, 179

Douglas, G.W. and M. Ryan. Conservation Evaluation of the Prairie Lupine, Lupinus lepidus var. lepidus, in Canada, 147

Douglas, G.W. and M. Ryan. Conservation Evaluation of the Seaside Birds-foot Trefoil, Lotus formosissimus, in Canada, 153

Douglas, G.W., J.L. Penny, and K. Barton. Conservation Evaluation of Stoloniferous Pussytoes, Antennaria flagellaris, in Canada, 183

Douglas, G.W., J.L. Penny, and K. Barton. Conservation Evaluation of the Pacific Population of Dwarf Woollyheads, Psilocarphus brevissimus var. brevissimus, in Canada, 163

Douglas, H., 15

Douglas 1938 - 2005, A Tribute to George Wayne, 135

Douglas-fir, 148,158,164,169,176,184,348 Coastal, 180

Dove, Mourning, 294 Rock, 294

Dragonflies (Odonata) in the Baie des Chaleurs Region of Quebec and New Brunswick in Late Summer and Autumn, Use of Saltmarsh by, 413

Dragonfly, 347

Dragonfly Cordulegaster dorsalis (Odonata: Cordulegastridae) in an Ephemeral Habitat in Southwestern British Columbia, Life History Phenology and Sediment Size Association of the, 347

Dragonlet, Seaside, 417

Drosera intermedia, 354

Dryocopus pileatus, 299

Duck, 2

Common Eider, 236

Ring-necked, 6

Wood, 236,303

Duck Nests in Saskatchewan, Summer Movements and Impact of Individual Striped Skunks, Mephitis mephitis, on, 342

Ducks, Ring-billed Gull, Larus delawerensis, Food Piracy on Diving, 109

Duffe, J., 289

Eagle, Bald, 109,237,477

Eagle, Haliaeetus leucocephalus, Preying on Maritime Garter Snake, Thamnophis sirtalis pallidulus, on Cape Breton Island, Nova Scotia, Bald, 477

Eatough, D.L., 474,478

Eel, American, 477

Editor's Report for Volume 119, 260

Eider, Common, 233

Eider, Somateria mollissima, Duckling, Osprey, Pandion haliaetus, Depredates Common, 236

Elk, 110,188,314

Elk, Cervus elaphus, Calf Observed Near Bancroft, Ontario, Late-born, 188

Elk, Cervus elaphus, Carcass from a Small Lake in Riding Mountain National Park, Manitoba, Probable Black Bear, Ursus americana, Retrieval of an, 110

Elymus canadensis, 336

lanceolatus ssp. lanceolatus, 336

repens, 355

trachycaulus, 184
Emerald, Kennedy's, 417

Petite, 417

Ski-tipped, 417

Emphoropsis, 180

Enallagma aspersum, 417

civile, 413

durum, 417

ebrium, 416

hageni, 415

Enteromorpha sp., 417

Epigaea repens, 355

Epilobium angustifolium, 171

densiflorum, 160

Épinettes, 238

Epistrophe nitidicollis, 17

Eptatretus stoutii, 363

Eptatretus stoutii, Spotted Ratfish, Hydrolagus colliei, and Scavenger Activity on Tethered Carrion in Subtidal Benthic Communities off Western Vancouver Island, Pacific Hagfish, 363

Eptesicus fuscus, 39

Equus cabalus, 314

Ericameria nauseosus var. speciosa, 184

Erigeron, 137

compositus var. glabratus, 184

leibergii, 137

salishii, 137

strigosus, 355

Eriogonum heracleoides var. angustifolium, 184 ovalifolium var. nivale, 184

Eristalis dimidiatus, 17

Erophila verna, 320

Erpobdella, 449

Erskine, A.J. Recent Declines of House Sparrows, Passer domesticus, in Canada's Maritime Provinces, 43

Erythrodiplax berenice, 413

Esox lucius, 421,481

Eubalaena glacialis, 95

Eupeodes americanus, 17 perplexus, 17 pomus, 17

Euphausiid, 96

Euphorbia esula var. esula, 337

Fairybells, Hooker's, 171

Falco columbarius, 46 sparverius, 290,303

Falsebox, 169

Fargey, P., Review by, 382

Feathermoss, 169

Felis catus, 46 concolor, 314

Fern, Bracken, 172 Maidenhair, 138

Fescue, 226

Festuca spp., 226 filiformis, 355 trachyphylla, 355

Fir, Amabilis, 172 Balsam, 50,100,236,299,433,440

Douglas, 11

Subalpine, 100

Fireweed, 171

Fisher, 62,101,229

Flax, 343

Fleabane, 137 
Leiberg's, 137

Flicker, Northern, 294,302

Forktail, Eastern, 417

Fox, Arctic, 36

Red, 36,51,68,263,342,468

San Joaquin Kit, 52,210

Santa Cruz Island, 210

Swift, 31,52

Foxes, Vulpes velox, in Southeastern Colorado, Multiple Scale Den Site Selection by Swift, 31

Foxglove, Middleton False, 357

Fragaria virginiana, 355

Freedman, B., 298

Frog, Boreal Chorus, 87

Columbia Spotted, 87

Green, 294

Mink, 239

Western Spotted, 87

Wood, 87

Frog, Rana septentrionalis, in Southeastern Labrador, The Mink, 239

Frostweed, Long-Branch, 359

Fucus vesiculosus, 236

Fulmar, 458

Fulmarus glacialis, 458

Gale, Sweet, 172,240

Galium aparine, 180

Gallant, D., 438

Galvez, I., 241

Gambusia affinis, 421

Gasterosteus aculeatus, 240,422

Gastromyzon sp., 424

Gaultheria ovatifolia, 171 procumbens, 355 shallon, 11,172

Gawn, J., Reviews by, 490,499

Gawn, M., Reviews by, 119,379

Gaylussacia baccata, 355 dumosa, 360

Geese, Chen caerulescens caerulescens, Exceeding the Carrying Capacity of the Fraser River Delta's Brackish Marshes?, Are Lesser Snow, 213

Gese, E.M., 31

Gilhen, J., 241

Glider, Wandering, 417

Globicephala melas, 97

Glycine max, 226

Gnaphalium palustre, 164

Goat, Mountain, 314

Golden-aster, Hairy, 336

Goldeneye, Common, 5,109

Goldenrod, 226

Goldeye, 422

Goldfinch, American, 294

Goldfish, 422

Goldsborough, L.G., 83

Gomphid, 350

Good King Henry, 340

Goodwin, C.E., 319

Goodwin, C.E., Review by, 379

Goose spp., 5

Canada, 109

Lesser Snow, 213

White, 214

Goosefoot, Fremont's, 340
Narrow-leaved, 336

Smooth, 335

Goosefoot, Chenopodium subglabrum (Chenopodiaceae), in

Canada, A Conservation Evaluation of Smooth, 335

Gossypium spp., 226

Gowans, S., 93

Grackle, Common, 294

Grass, Orchard, 154,160

Sand, 336

Switch, 226

Grayling, Arctic, 422

Grebe, 2

Horned, 109

Red-necked, 109

Greenling, Kelp, 363

Griffiths, A., 363

Grouseberry, 172

Grus canadensis, 68

Gull, 2

Bonaparte's, 5

Ring-billed, 109

Gull, Larus delawerensis, Food Piracy on Diving Ducks, Ring-billed, 109

Gulo gulo, 111

Gum, Sweet, 226

Guppy, 422

Haemopis, 443

caeca, 450

grandis, 443

kingi, 444

lateromaculata, 443

marmorata, 443

plumbea, 447

sanguisuga, 444

septagon, 444

terrestris, 447

Haemopis lateromaculata (Hirudinea: Haemopidae): Its North America Distribution and Additional Notes on Species Description, The Leech, 443

Hagfish, Pacific, 363

Hagfish, Eptatretus stoutii, Spotted Ratfish, Hydrolagus colliei, and Scavenger Activity on Tethered Carrion in Subtidal Benthic Communities off Western Vancouver Island, Pacific, 363

Hairgrass, Annual, 164

Early, 154,320

Silvery, 320

Haliaeetus leucocephalus, 109,237,477

Haliaeetus leucocephalus, Preying on Maritime Garter Snake, Thamnophis sirtalis pallidulus, on Cape Breton Island, Nova Scotia, Bald Eagle, 477

Hall, M.N., 57

Hamr, J., 57

Hanke, G.F., M.C.E. McNall, and J. Roberts. First Records of the Yellow Bullhead, Ameiurus natalis, a Loricariid Catfish, Panaque suttonorum, and a Silver Pacu, Piaractus cf. P. brachypomus, in British Columbia, 421

Hare, Arctic, 51,466 Snowshoe, 51,68,205

Harrier, Northern, 109

Harrington, F.H. Double Marking in Arctic Wolves, Canis lupus arctos: Influence of Order on Posture, 471

Haskell, S.P. First Record of a River Otter, Lontra canadensis, Captured on the Northeastern Coast of Alaska, 235 
Hawk, Sharp-shinned, 46

Hawkweed, White, 170

Hazelnut, Beaked, 170

Hearn, B.J., J.T. Neville, W.J. Curran, and D.P. Snow. First Record of the Southern Red-Backed Vole, Clethrionomys gapperi, in Newfoundland: Implications for the Endangered Newfoundland Marten, Martes americana atrata, 50

Hedge-parsley, Upright, 180

Heide-Jørgensen, M.P., 457

Helianthemum canadense, 355

Helianthus petiolaris ssp. petiolaris, 336

Helophilus fasciatus, 17

Hemlock, Coastal Western, 172

Eastern, 290

Western, 11,170,348

Herring, 96

Heterotheca villosa var. villosa, 336

Hexagrammos decagrammus, 363

Hickory, 226

Hieracium albiflorum, 170 pilosella, 355 piloselloides, 355

Hiodon alosoides, 422

Holcus lanatus, 154

Holmes, R.B., 403

Holodiscus discolor, 158,171

Horse, Wild, 314

Houston, A.E., 225,452

Houston, C.S., Reviews by, 124,382,385,496,497,498

Hoverfly, 15

Hovingh, P. The Leech Haemopis lateromaculata (Hirudinea: Haemopidae): Its North America Distribution and Additional Notes on Species Description, 443

Huckleberry, 348,360 Red, 11,172

Hudsonia ericoides, 355

Huettmann, F., Reviews by, 125,254,491

Hutchinson, R., 413

Hwang, Y.T. and S. Larivière. A Test of Interspecific Effects of Introduced Eastern Grey Squirrels, Sciurus carolinensis, on Douglas's Squirrels, Tamiasciurus douglasii, in Vancouver, British Columbia, 10

Hydrolagus colliei, 363

Hydrolagus colliei, and Scavenger Activity on Tethered Carrion in Subtidal Benthic Communities off Western Vancouver Island, Pacific Hagfish, Eptatretus stoutii, Spotted Ratfish, 363

Hyena, Spotted, 52

Hylaeus elliptica, 19

Hylocomium splendens, 171

Hymenoptera, 295

Hypericum perforatum, 355

Hypochaeris radicata, 154

Hypophthalmichthys sp., 424

Hypostomus, 424

Ictalurus furcatus, 424 punctatus, 422

Ischnura verticalis, 416

Isoetes nuttallii, 160

Jackal, 263

Jay, Blue, 294
Jennings, J.B., M.L. Kennedy, A.E. Houston, and B.D. Carver. Predation on Artificial Nests of Northern Bobwhites, Colinus virginianus, by Mammalian Mesopredators: Does the Problem-Individual Paradigm Fit?, 452

John, R., Reviews by, 113,114,119,123,244,251,255,381,386, $388,389,483,484,488,489$

Johnson, G., 298

Junco hyemalis, 294

Junco, Dark-eyed, 294

Juncus balticus, 417

effusus, 355

gerardii, 417

Juneberry, 360

Jung, T.S., A.M. Runck, D.W. Nagorsen, B.G. Slough, and T. Powell. First Records of the Southern Red-backed Vole, Myodes gapperi, in the Yukon, 331

Jung, T.S., B.G. Slough, D.W. Nagorsen, T.A. Dewey, and T. Powell. First Records of the Northern Long-eared Bat, Myotis septentrionalis, in the Yukon Territory, 39

Juniper, 32

Creeping, 336

Juniperus communis var. depressa, 355

horizontalis, 336

monosperma, 32

Kallemeyn, L.W., 71

Kalmia, 240

angustifolia, 355

Kenn, S., 438

Kennedy, A., 233

Kennedy, M.L., 225,452

Kestrel, 302 American, 290,303

Kindbergia oregana, 180

Kinnikinnick, 172

Kirk, D.A., 298

Kitchen, A.M., E.M. Gese, and S.G. Lupis. Multiple Scale Den Site Selection by Swift Foxes, Vulpes velox, in Southeastern Colorado, 31

Kneteman, J., 313

Knotweed, Close-flowered, 164 Common, 164

Kogia breviceps, 97

Koi, 424

Kudzu, 226

Kuzyk, G.W., J. Kneteman, and F.K.A. Schmiegelow. Pack Size of Wolves, Canis lupus, on Caribou, Rangifer tarandus, Winter Ranges in Westcentral Alberta, 313

l'Umbre de vase, 238

l'Umbre de vase, Umbra limi, dans le nord-est du Québec, Extension de l'aire de distribution de, 238

Labrador, The Mink Frog, Rana septentrionalis, in Southeastern, 239

Lactuca canadensis var. longifolia, 355

Lagenorhynchus acutus, 95

albirostris, 95

obscurus, 97

Laidre, K.L., M.P. Heide-Jørgensen, and J.R. Orr. Reactions of Narwhals, Monodon monoceros, to Killer Whale, Orcinus orca, Attacks in the Eastern Canadian Arctic, 457 
Lamb's-quarters, 337

Lambdina fiscellaria fiscellaria, 50

Landriault, L.J., M.N. Hall, J. Hamr, and F.F. Mallory. Longrange Homing by an Adult Female Black Bear, Ursus americanus, 57

Larch, American, 433

Larivée, J., 289

Larivière, S., 10

Larivière, S., L.R. Walton, and F. Messier. Summer Movements and Impact of Individual Striped Skunks, Mephitis mephitis, on Duck Nests in Saskatchewan, 342

Larix laricina, 314,355,433

Larkspur, Menzies, 154

Larus delawerensis, 109 philadelphia, 5

Larus delawerensis, Food Piracy on Diving Ducks, Ringbilled Gull, 109

Lasioglossum admirandus, 19

divergens, 19

laevissimus, 19

nymphaearum, 19

quebecensis, 19

rohweri, 19

versans, 19

zephyrus, 19

Lasionycteris noctivagans, 39

Latissimus dorsi, 406

Lauff, R., Reviews by, 118,121,243,380

Lawlor, J.L., 93

Leatherleaf, 23

Lechea intermedia, 355

intermedia var. intermedia, 361

intermedia var. juniperina, 361

Ledum groenlandicum, 172

Leech, 443

Leech Haemopis lateromaculata (Hirudinea: Haemopidae): Its North America Distribution and Additional Notes on Species Description, The, 443

Lejota aerea, 17 cyanea, 17

Lemming, 466

Lentil, 343

Leontodon autumnalis, 355

Lepeophtheirus salmonis, 199

Lepeophtheirus salmonis Infestation Levels on Juvenile Wild Pink, Oncorhynchus gorbuscha, and Chum, O. keta, Salmon to Arrival of Parasitized Wild Adult Pink Salmon, Response of the Sea Louse, 199

Lepidosetta bilineata, 364

Lepisosteus oculatus, 424 osseus, 423

platostomus, 424

Lepomis gibbosus, 422

macrochirus, 422

Lepus americanus, 68,205

americanus struthopus, 51

arcticus, 466

arcticus bangsii, 51

Lestes congener, 415 disjunctus, 415

forcipatus, 416

inaequalis, 417

rectangularis, 417

unguiculatus, 417

Leucanthemum vulgare, 355
Leucorrhinia frigida, 417

Libellula luctuosa, 417

pulchella, 417

quadrimaculata, 415

Lichen, 172

Limonius aeger, 19 confusus, 19

Linaria canadensis, 355 genistifolia ssp. dalmatica, 176

Lindell, J., Review by, 252

Linnaea borealis, 172

Lion, 52

Liquidambar styraciflua, 226

Liriodendron tulipifera, 226

Listera cordata, 170

Liu, P.S., 225

Lobelia dortmanna, 240

Lobelia, Water, 240

Lomatium ambiguum, 184

Lontra canadensis, 235 canadensis degener, 51

Lontra canadensis, Captured on the Northeastern Coast of Alaska, First Record of a River Otter, 235

Loon, 2

Looper, Hemlock, 50

Loosestrife, Purple, 307

Lota lota, 422,480

Lotus corniculatus, 157 formosissimus, 153,157 pinnatus, 153,157

Lotus formosissimus, in Canada, Conservation Evaluation of the Seaside Birds-foot Trefoil, 153

Lotus pinnatus, in Canada, Conservation Evaluation of the Bog Bird's-foot Trefoil, 157

Louse, Sea, 199

Louse Lepeophtheirus salmonis Infestation Levels on Juvenile Wild Pink, Oncorhynchus gorbuscha, and Chum, $O$. keta, Salmon to Arrival of Parasitized Wild Adult Pink Salmon, Response of the Sea, 199

Lumpsucker, 464

Lupine, Prairie, 147 Silky, 176

Lupine, Lupinus lepidus var. lepidus, in Canada, Conservation Evaluation of the Prairie, 147

Lupinus lepidus, 147

lepidus var. lepidus, 147

lepidus var. lobbii, 147

lyallii, 147

minimus, 147

sericeus, 176

Lupinus lepidus var. lepidus, in Canada, Conservation Evaluation of the Prairie Lupine, 147

Lupis, S.G., 31

Lutra canadensis, 62

Luzula multiflora, 355

Lycaena dospassosi, 419

Lycaon pictus, 52

Lycopodium dendroideum, 355

obscurum, 357

tristachyum, 355

Lygodesmia juncea, 336

Lynx lynx susolanus, 51 pardinus, 229

rufus, 230

Lynx, Canada, 51

Iberian, 229 
Lysichitum americanum, 172

Lythrum salicaria, 307

MacKinnon, C. and A. Kennedy. An Observation of the Spring 2006 Migration of Black Scoter Melanitta nigra, in Northumberland Strait, Interrupted by the Confederation Bridge, New Brunswick - Prince Edward Island, 233

Madtom, Tadpole, 424

Mahonia nervosa, 171

Maianthemum canadense, 355

Maine, Lady Crabs, Ovalipes ocellatus, in the Gulf of. 106

Malacosoma disstria, 433

Mallard, 6

Mallon, J.C. and R.B. Holmes. A Reevaluation of Sexual Dimorphism in the Postcranium of the Chasmosaurine Ceratopsid Chasmosaurus belli (Dinosauria: Ornithischia), 403

Mallory, F.F., 57

Malus pumila, 355

Manitoba, Northern Range Expansion and Invasion by the Common Carp, Cyprinus carpio, of the Churchill River System in, 83

Manitoba, Probable Black Bear, Ursus americana, Retrieval of an Elk, Cervus elaphus, Carcass from a Small Lake in Riding Mountain National Park, 110

Maple, 226

$$
\text { Big Leaf, 11,180 }
$$

Douglas, 171

Red, 299

Sugar, 299

Vine, $11,171,348$

Marczak, L.B., J.S. Richardson, and M.-C. Classen. Life History Phenology and Sediment Size Association of the Dragonfly Cordulegaster dorsalis (Odonata: Cordulegastridae) in an Ephemeral Habitat in Southwestern British Columbia, 347

Marine Turtle Newsletter, 132,258,392,502

Marten, 62

American, 100

Newfoundland, 50

Pine, 52

Marten, Martes americana atrata, First Record of the Southern Red-Backed Vole, Clethrionomys gapperi, in Newfoundland: Implications for the Endangered Newfoundland, 50

Marten, Martes americana, in Western Alberta Boreal Forests, Winter Habitat Use by American, 100

Martes americana, 62,100

americana atrata, 50

martes, 52

pennanti, 62,101,229

Martes americana atrata, First Record of the Southern Red-Backed Vole, Clethrionomys gapperi, in Newfoundland: Implications for the Endangered Newfoundland Marten, 50

Martes americana, in Western Alberta Boreal Forests, Winter Habitat Use by American Marten, 100

Mary, Blue-eyed, 180

Maunder, J.E., 239

McCarthy, J., Review by, 387

McCorquodale, D., Review by, 486

McNall, M.C.E., 421

Meadow-foxtail, Carolina, 164

Meadowhawk, Autumn, 417

Band-winged, 417
Black, 417

Cherry-faced, 417

Saffron-winged, 417

White-faced, 417

Mech, L.D. Urine-marking and Ground-scratching by Freeranging Arctic Wolves, Canis lupus arctos, in Summer, 466

Meganyctiphanes norvegica, 96

Megaptera novaeangliae, 95

Megascops asio, 289

Megascops asio, Population in an Apple-Producing Region of Southern Quebec, First Observations of an Eastern Screech-Owl, 289

Meikle, D.B., 192

Melampyrum lineare, 357 lineare var. lineare, 170

Melanitta spp., 232 nigra, 233

Melanitta nigra, in Northumberland Strait, Interrupted by the Confederation Bridge, New Brunswick - Prince Edward Island, An Observation of the Spring 2006 Migration of Black Scoter, 233

Melanitta spp., Migrations Interrupted by Confederation Bridge: An Update, Scoter, 232

Melanostoma, 19 mellinum, 17

Melanotus castanipes, 19

Melanpyrum lineare, 355

Meles meles, 52

Melic, Harford's, 180

Melica harfordii, 180

Melilotus spp., 337

Meliscaeva cinctellam 17

Mennell, R.L., 87

Mephitis mephitis, 229,342,452

Mephitis mephitis, on Duck Nests in Saskatchewan, Summer Movements and Impact of Individual Striped Skunks, 342

Merganser, Red-breasted, 109

Mergus serrator, 109

Merlin, 46

Messier, F., 342

Metynnis sp., 424

Mice, Peromyscus leucopus: The Effects of Forest Patch Size, Edge and Surrounding Vegetation Type, Annual Variation in Habitat Use by White-footed, 192

Micropterus dolomieu, 421 salmoides, 422

Microtus sp., 52,291 pennsylvanicus, 264,294,332,436 pennsylvanicus terraenovae, 50

Milk-vetch, Timber, 176

Mimulus guttatus, 158

Mineau, P., 289

Miner's-lettuce, 180

Mink, 51,62

Minnesota and Ontario, Lake Sturgeon, Acipenser fulvescens, Movements in Rainy Lake, 71

Minnow, Fathead, 421

Misgurnus anguillicaudatus, 424

Mitchell, S., Review by, 490

Mitrow, G., 428

Mollibdella, 444 grandis, 444

Mollugo verticillata, 355

Molothrus ater, 294 
Monkey-flower, Yellow, 158

Monoclonius belli, 403

Monodon monoceros, 457

Monodon monoceros, to Killer Whale, Orcinus orca, Attacks in the Eastern Canadian Arctic, Reactions of Narwhals, 457

Monopterus sp., 424

Monotropa hypopithys, 355

Montia parvifolia, 158

Montia, Small-leaved, 158

Mooreobdella, 449

Moose, 51,205,313,367

Morone saxatilis, 422

Morton, A. and R. Williams. Response of the Sea Louse Lepeophtheirus salmonis Infestation Levels on Juvenile Wild Pink, Oncorhynchus gorbuscha, and Chum, O. keta, Salmon to Arrival of Parasitized Wild Adult Pink Salmon, 199

Mosquitofish, 421

Western, 422

Moss, Heron's-bill, 180

Lanky, 171

Oregon Beaked, 180

Step, 171

Mouse, Deer, 50,57,332

House, 51

Meadow Jumping, 332

White-footed, 192

Mousetail, Tiny, 164

Mus musculus domesticus, 51

Muskox, 466

Muskrat, 51

Mustela ermina, 62

erminea richardsonii, 51

vison, 51,62

Myleus sp., 424

Myodes californicus, 332 gapperi, 331

glareolus, 332

rufocanus, 332 rutilus, 331

Myodes gapperi, in the Yukon, First Records of the Southern Red-backed Vole, 331

Myosotis stricta, 320

Myosurus minimus, 164

Myotis evotis, 39

keenii, 40

lucifugus, 39

lucifugus lucifugus, 51

septentrionalis, 39,51,332

volans, 39

Myotis septentrionalis, in the Yukon Territory, First Records of the Northern Long-eared Bat, 39

Myoxocephalus thompsonii, 480

Myoxocephalus thompsonii, in Northeastern Alberta, A New Record of Deepwater Sculpin, 480

Myrica gale, 172,240 pensylvanica, 355

Myxocyprinus asiaticus, 424

Nagorsen, D.W., 39,331

Narwhal, 457

Narwhals, Monodon monoceros, to Killer Whale, Orcinus orca, Attacks in the Eastern Canadian Arctic, Reactions of, 457

Neely, D.A., 480
Neoascia distincta, 17

Neohypdonus tumescens, 19

Nephelopsis, 449

Neuhold, J., 188

Neville, J.T., 50

New Brunswick, Eastern Canada, MtDNA Analyses on Hair Samples Confirm Cougar, Puma concolor, Presence in Southern, 438

New Brunswick, Effects of Timber Harvesting and Plantation Development on Cavity-nesting Birds in, 298

New Brunswick in Late Summer and Autumn, Use of Saltmarsh by Dragonflies (Odonata) in the Baie des Chaleurs Region of Quebec and, 413

New Brunswick - Prince Edward Island, An Observation of the Spring 2006 Migration of Black Scoter Melanitta nigra, in Northumberland Strait, Interrupted by the Confederation Bridge, 233

Newfoundland: Implications for the Endangered Newfoundland Marten, Martes americana atrata, First Record of the Southern Red-Backed Vole, Clethrionomys gapperi, in, 50

Newfoundland Marten, Martes americana atrata, First Record of the Southern Red-Backed Vole, Clethrionomys gapperi, in Newfoundland: Implications for the Endangered, 50

Newt, Roughskin, 90

Nine-bark, 158

Noel, L.E., M.K. Butcher, M.A. Cronin, and B. Streever. Assessment of Effects of an Oil Pipeline on Caribou, Rangifer tarandus granti, Use of Riparian Habitats in Arctic Alaska, 2001-2003, 323

Nol, E., H. Douglas, and W.J. Crins. Responses of Syrphids, Elaterids and Bees to Single-tree Selection Harvesting in Algonquin Provincial Park, Ontario, 15

North America Distribution and Additional Notes on Species Description, The Leech Haemopis lateromaculata (Hirudinea: Haemopidae): Its, 443

Northwest Territories, Canada, Extension of Coyote, Canis latrans, Breeding Range in the, 67

Notropis hudsonius, 422

Noturus gyrinus, 424

Nova Scotia, An Analysis of the Vascular Flora of Annapolis Heathlands, 351

Nova Scotia, Bald Eagle, Haliaeetus leucocephalus, Preying on Maritime Garter Snake, Thamnophis sirtalis pallidulus, on Cape Breton Island, 477

Nova Scotia, Temporal Variability of Cetaceans near Halifax, 93

Novakowski 1925-2004, A Tribute to Nicholas Stephen, 370

Nuphar variegata, 240

Nuthatch, 290

Red-breasted, 302

White-breasted, 302

O’Neill, J., Reviews by, 120,485,487,498

Oak, 226

Oat, 343

Garry, $150,154,157,180$

Oatgrass, One-spike, 164,184

Oceanspray, 171

Odocoileus sp., 68

heminous, 40,314

hemionus sitkensis, 61

virginianus, 29,40,64,68,209,313,367

Oldham, M.J., C.E. Goodwin, and S. Blaney. Bur Buttercup, Ranunculus testiculatus, New to Eastern Canada, 319 
Olson, S.L. Bald Eagle, Haliaeetus leucocephalus, Preying on Maritime Garter Snake, Thamnophis sirtalis pallidulus, on Cape Breton Island, Nova Scotia, 477

Omble de fontaine, 238

Oncorhynchus clarki lewisi, 421

gorbuscha, 199,421

keta, 199,421

kisutch, 421

mykiss, 90,421

mykiss aguabonita, 422

nerka, 421

tshawytscha, 421

Oncorhynchus gorbuscha, and Chum, O. keta, Salmon to Arrival of Parasitized Wild Adult Pink Salmon, Response of the Sea Louse Lepeophtheirus salmonis Infestation Levels on Juvenile Wild Pink, 199

Oncorhynchus keta, Salmon to Arrival of Parasitized Wild Adult Pink Salmon, Response of the Sea Louse Lepeophtheirus salmonis Infestation Levels on Juvenile Wild Pink, Oncorhynchus gorbuscha, and Chum, 199

Ondatra zibethicus obscurus, 51

Onion, Nodding, 154 Slim-leaf, 160

Ontario, A New Record Size Wolf, Canis lupus, Group for, 367

Ontario, in 2001, Observations on the Nesting of the Northern Hawk Owl, Surnia ulula, near Timmins and Iroquois Falls, Northeastern, 433

Ontario, Lake Sturgeon, Acipenser fulvescens, Movements in Rainy Lake, Minnesota and, 71

Ontario, Late-born Elk, Cervus elaphus, Calf Observed Near Bancroft, 188

Ontario Natural Heritage Information Centre Science and Information Newsletter 11(1) Winter 2006, 132

Ontario, Responses of Syrphids, Elaterids and Bees to Single-tree Selection Harvesting in Algonquin Provincial Park, 15

Ontario, with Some Notes on Nesting Behaviour, New Nesting Records of the Le Conte's Sparrow, Ammospiza leconteii, from Northeastern, 22

Onychogomphus uncatus, 350

Opossum, Virginia, 229,452

Orcinus orca, 457

Orcinus orca, Attacks in the Eastern Canadian Arctic, Reactions of Narwhals, Monodon monoceros, to Killer Whale, 457

Oreamnos americanus, 314

Oregon-grape, Dull, 17

Oreochromis sp., 424

Orr, J.R., 457

Orthasterias koehleri, 364

Orthocarpus barbatus, 137

Oryzopsis asperifolia, 358

Osmia, 180

atriventris, 19

Osprey, 236

Osprey, Pandion haliaetus, Depredates Common Eider, Somateria mollissima, Duckling, 236

Ottawa Field-Naturalists' Club Awards for 2005, The, 504

Ottawa Field-Naturalists' Club 10 January 2006, Minutes of the 127th Annual Business Meeting of The, 393

Otter, 5

River, 62,235

Otter, Lontra canadensis, Captured on the Northeastern Coast of Alaska, First Record of a River, 235
Ovalipes ocellatus, 106

Ovalipes ocellatus, in the Gulf of Maine, Lady Crabs, 106

Ovibos moschatus, 466

Ovis canadensis, 314 dalli, 205

Owl, Barred, 290,302,

Great Horned, 290

Long-eared, 295

Northern Hawk, 433

Northern Saw-whet, 294,303 Saw-whet, 302

Owl, Surnia ulula, near Timmins and Iroquois Falls, Northeastern Ontario, in 2001, Observations on the Nesting of the Northern Hawk, 433

Owl-clover, Grand Coulee, 137

Ozotocerus bezarticus, 64

Pacu, Red-bellied, 421

Silver, 421

Pacu, Piaractus cf. P. brachypomus, in British Columbia, First Records of the Yellow Bullhead, Ameiurus natalis, a Loricariid Catfish, Panaque suttonorum, and a Silver, 421

Pan troglodytes, 264

Panaque sp., 424

nigrolineatus, 424

suttonorum, 421

Panaque, Blue-eyed, 423 Royal, 424

Panaque suttonorum, and a Silver Pacu, Piaractus cf. P. brachypomus, in British Columbia, First Records of the Yellow Bullhead, Ameiurus natalis, a Loricariid Catfish, 421

Pandalus platyceros, 364

Pandion haliaetus, 236

Pandion haliaetus, Depredates Common Eider, Somateria mollissima, Duckling, Osprey, 236

Panicum virgatum, 226

Pantala spp., 418 flavescens, 416

Panthera leo, 52

Paquet, P.C., 61

Parasyrphus sp., 17 semiinterruptus, 17

Passer domesticus, 43,264

Passer domesticus, in Canada's Maritime Provinces, Recent Declines of House Sparrows, 43

Patrikeev, M. New Nesting Records of the Le Conte's Sparrow, Ammospiza leconteii, from Northeastern Ontario, with Some Notes on Nesting Behaviour, 22

Patrikeev, M. Observations on the Nesting of the Northern Hawk Owl, Surnia ulula, near Timmins and Iroquois Falls, Northeastern Ontario, in 2001, 433

Paxistima myrsinites, 169

Pea, 343

Pennant, Calico, 417

Penny, G., Reviews by, 117,246

Penny, J.L., 163,175,179,183

Penny, J.L. A Tribute to George Wayne Douglas 1938 - 2005, 135

Pentaceratops, 403

Perca flavescens, 422

Perch, Yellow, 422

Percopsis omiscomaycus, 422

Percymoorensis, 444 
caballeroi, 444

kingi, 444

lateromaculata, 444

marmorata, 444

terrestris, 444

Peromyscus sp., 294

leucopus, 192

maniculatus, 50,57,332

Peromyscus leucopus: The Effects of Forest Patch Size, Edge and Surrounding Vegetation Type, Annual Variation in Habitat Use by White-footed Mice, 192

Phacelia linearis, 176

Phacelia, Thread-leaved, 176

Phenacomys ungava, 332

Phoca spp., 466

hispida, 464

vitulina, 62

Phocoena phocoena, 95

Phoebe, Eastern, 294

Phragmites australis, 307

australis subspecies americanus, 307 australis subspecies australis, 307

Phragmites australis subspecies australis, in the Southern Ottawa District, Recent Invasion, Current Status and Invasion Pathway of European Common Reed, 307

Physalis alkekengi, 355

Physocarpus capitatus, 158

Piaractus sp., 424 brachypomus, 421

Piaractus brachypomus, in British Columbia, First Records of the Yellow Bullhead, Ameiurus natalis, a Loricariid Catfish, Panaque suttonorum, and a Silver Pacu, Piaractus cf., 421

Piaractus cf. P. brachypomus, in British Columbia, First Records of the Yellow Bullhead, Ameiurus natalis, a Loricariid Catfish, Panaque suttonorum, and a Silver Pacu, 421

Picard, I., 239

Picea sp., 20,110,236,238,431

abies, 299

engelmannii, 100

glauca, 100,299,314,331,355

mariana, 22,100,240,299,314,331,355,433

rubens, 299,355,440

sitchensis, 11

Picoides arcticus, 303

pubescens, 303

villosus, 294,303

Pigeon, 57

Pike, Northern, 421,480

Pimephales promelas, 421

Pine, 290

Jack, 299,433

Loblolly, 226

Lodgepole, 100,148,170,314

New Jersey, 351

Pinyon, 32

Ponderosa, 164,176,184

Red, 351

Scots, 320

Western White, 172

White, 20

Pinus sp., 290

banksiana, 299,355,433

contorta, 100,170,314

contorta var. contorta, 148 edulis, 32

monticola, 172

ponderosa, 164,176,184

resinosa, 351

strobus, 20,355

sylvestris, 298,320,355

taeda, 226

Pipiza femoralis, 17

Piranha, 421

Plagiobothrys scouleri, 158,164

Plantago lanceolata, 154

Plantain, Ribwort, 154

Plathemis lydia, 417

Platycheirus, 19

confusus, 17

obscurus, 17

Platygobio gracilis, 422

Pleco, Royal, 424

Plectritis congesta, 154,158

Pleurozium schreberi, 169,358

Роа аппиа, 320

bulbosa, 320

compressa, 356

pratensis, 160,356

Podiceps auritus, 109 grisegena, 109

Poecile atricapillus, 294,303

hudsonicas, 303

Poecilia reticulata, 422

Polygala sanguinea, 357

Polygonella articulata, 360

Polygonum sp., 356 aviculare, 164 polygaloides ssp. confertiflorum, 164

Polytrichum commune, 358 juniperinum, 358

Pomoxis nigromaculatus, 422

Pondlily, Yellow, 240

Popcornflower, Scouler's, 158,164

Poplar, 433

Balsam, 22

Tulip, 226

Populus, 428,433

balsamifera, 22

deltoides, 226

grandidentata, 356

tremuloides, 22,100,314,343,356

Porpoise, Harbour, 95

Potamogeton pectinatus, 416

Potentilla argentea, 356

canadensis, 356

pacifica, 214

recta, 356

simplex, 356

Poulin, R., Reviews by, 128,253

Powell, T., 339,331

Prairie Dog, 36 Black-tailed, 31

Primrose, Dense-spike Evening, 160

Prince Edward Island, An Observation of the Spring 2006 Migration of Black Scoter Melanitta nigra, in Northumberland Strait, Interrupted by the Confederation Bridge, New Brunswick -, 233

Procyon lotor, 57,225,290,342,452

Procyon lotor, Occurrence Through the Use of Microhabitat Variables, Predicting Raccoon, 225 
Pronghorn, 210

Prosartes hookeri var. oregana, 171

Proulx, G. Winter Habitat Use by American Marten, Martes americana, in Western Alberta Boreal Forests, 100

Prunus pensylvanica, 50,356 serotina, 356

susquehanae, 360 virginiana, 356

Pseudacris maculata, 88 septentrionalis, 90 triseriata maculata, 88 triseriata septentrionalis, 88

Pseudoroegneria spicata, 176

Pseudotsuga menziesii, 11,148,158,164,169,176,180,184,348

Psilocarphus brevissimus var. brevissimus, 163 brevissimus var. multiflorus, 163 elatior, 163 tenellus var. tenellus, 163

Psilocarphus brevissimus var. brevissimus, in Canada, Conservation Evaluation of the Pacific Population of Dwarf Woolly-heads, 163

Psoralea, Lance-leaved, 336

Psoralidium lanceolatum, 336

Pteridium acquilinum var. latiusculum, 357 aquilinum, 172 aquilinum var. latiusculum, 356

Pueraria lobata, 226

Puma concolor, $40,438,476$ concolor couguar, 438

Puma concolor, Presence in Southern New Brunswick, Eastern Canada, MtDNA Analyses on Hair Samples Confirm Cougar, 438

Pumpkinseed, 422

Pussytoes, Stoloniferous, 183

Pussytoes, Antennaria flagellaris, in Canada, Conservation Evaluation of Stoloniferous, 183

Pycnopodia helianthoides, 364

Pygocentrus sp., 421 nattereri, 424

Pylodictis olivaris, 424

Quebec and New Brunswick in Late Summer and Autumn, Use of Saltmarsh by Dragonflies (Odonata) in the Baie des Chaleurs Region of, 413

Québec, Extension de l'aire de distribution de l'Umbre de vase, Umbra limi, dans le nord-est du, 238

Quebec, First Observations of an Eastern Screech-Owl, Megascops asio, Population in an Apple-Producing Region of Southern, 289

Quebec, Herpetological Survey 2006 Atlas of Amphibians and Reptiles of, 258

Quercus spp., 226 garryana, 150,154,157 rubra, 356

Quillwort, Nuttall's, 160

Quinoa, 340

Quiscalus quiscula, 294

Rabbit, Cottontail, 475

Rabbit-brush, Common, 184

Raccoon, 57,225,290,342,452

Raccoon, Procyon lotor, Occurrence Through the Use of Microhabitat Variables, Predicting, 225

Rana clamitans, 294

luteiventris, 88 septentrionalis, 239

sylvatica, 90

Rana septentrionalis, in Southeastern Labrador, The Mink Frog, 239

Rangifer tarandus, 313

tarandus caribou, 313,367

tarandus granti, 205,323

tarandus terraenovae, 51

Rangifer tarandus granti, Use of Riparian Habitats in Arctic Alaska, 2001-2003, Assessment of Effects of an Oil Pipeline on Caribou, 323

Rangifer tarandus, Winter Ranges in Westcentral Alberta, Pack Size of Wolves, Canis lupus, on Caribou, 313

Ranunculus falcatus, 319 testiculatus, 319

Ranunculus testiculatus, New to Eastern Canada, Bur Buttercup, 319

Raphanus raphinistrum, 356

Rat, 478

Brown, 264

Norway, 51,264,475

Rat Poison Kills a Pack of Eastern Coyotes, Canis latrans, in an Urban Area, 478

Ratfish, Spotted, 363

Ratfish, Hydrolagus colliei, and Scavenger Activity on Tethered Carrion in Subtidal Benthic Communities off Western Vancouver Island, Pacific Hagfish, Eptatretus stoutii, Spotted, 363

Rattus norvegicus, 264,475

norvegicus norvegicus, 51

Raven, Common, 109,110

Redcedar, Western, 11,170,348

Redhead, 109

Reed, Common, 307 European Common, 307

Reed, Phragmites australis subspecies australis, in the Southern Ottawa District, Recent Invasion, Current Status and Invasion Pathway of European Common, 307

Reimchen, T.E., 61,363

Rhingia nasica, 17

Rhizobium, 154,159

Rhodeus sp., 424

Rhododendron albiflorum, 169

californicum, 169

canadense, 356

lapponicum, 169

macrophyllum, 169

Rhododendron, Pacific, 169 White-flowered, 169

Rhododendron macrophyllum, in Canada, Conservation Evaluation of Pacific Rhododendron, 169

Rhododendron, Rhododendron macrophyllum, in Canada, Conservation Evaluation of Pacific, 169

Rhytidiadelphus loreus, 171

Rice-grass, Indian, 336

Richards, N.L., P. Mineau, D.M. Bird, P. Wery, J. Larivée, and J. Duffe. First Observations of an Eastern ScreechOwl, Megascops asio, Population in an Apple-Producing Region of Southern Quebec, 289

Richardson, J.S., 347

Richardsonius balteatus, 421

Ringlet, Maritime, 419

Roberts, J., 421

Robin, American, 294

Rocksole, 364 
Ronconi, R.A. Predicting Bird Oiling Events at Oil Sands Tailings Ponds and Assessing the Importance of Alternate Waterbodies for Waterfowl: a Preliminary Assessment, 1

Ronquil, Northern, 364

Ronquilus jordani, 364

Rosa carolina, 353 nutkana, 158 virginiana, 353

Rosatte, R.C. and J. Neuhold. Late-born Elk, Cervus elaphus, Calf Observed Near Bancroft, Ontario, 188

Rose, Nootka, 158

Rosebay, Lapland, 169

Rubus sp., 236 allegheniensis, 356 arenicola, 353 hispidus, 353 idaeus var. strigosus, 18 particeps, 359 parviflorus var. parviflorus, 171 spectabilis, 11,348 vermontanus, 356

Rudbeckia hirta, 356

Rumex acetosella, 356

Runck, A.M., 331

Ruppia maritima, 414

Russian-thistle, 337

Ryan, M., 147,153

Rye, 343

Canada Wild, 336

Sagebrush, Big, 164,184 Hoary, 336

Salal, 11,172

Salamander, Long-toed, 90 Northwestern, 90

Salix sp., 158,314 exigua ssp. interior, 336 humilis, 356

Salmo gairdneri, 199 salar, 199,413,422 trutta, 199,422 trutta trutta, 199

Salmon, Atlantic, 199,413,422 Chinook, 422

Chum, 199,422

Pacific, 421

Sockeye, 422

Wild Pink, 199

Salmon, Response of the Sea Louse Lepeophtheirus salmonis Infestation Levels on Juvenile Wild Pink, Oncorhynchus gorbuscha, and Chum, O. keta, Salmon to Arrival of Parasitized Wild Adult Pink, 199

Salmonberry, 11,348

Salmonella, 46

Salsola tragus, 337

Salvelinus alpinus, 199,235,422 confluentus, 422

fontinalis, 238,422

malma, 422

namaycush, 422,481

Sander vitreus, 421

Sandlance, 96

Sandwort, Thread-leaved, 184

Sapin baumier, 238
Sapsucker, Yellow-bellied, 302

Sarotherodon sp., 424

Saskatchewan and the First Saskatchewan Records of Amelanchier sanguinea, Regional Variation in Amelanchi$e r$ in the Whitewood area of Southeastern, 428

Saskatchewan Records of Amelanchier sanguinea, Regional Variation in Amelanchier in the Whitewood area of Southeastern Saskatchewan and the First, 428

Saskatchewan, Summer Movements and Impact of Individual Striped Skunks, Mephitis mephitis, on Duck Nests in, 342

Saskatoon, 176

Sayornis phoebe, 294

Scaup, 6

Greater, 109

Lesser, 5

Schaefer, R.L., 192

Schmiegelow, F.K.A., 313

Scirpus sp., 214,295,417 americanus, 213

maritimus, 214

paludosus, 215

Sciurus carolinensis, 10,264 vulgaris, 10

Sciurus carolinensis, on Douglas's Squirrels, Tamiasciurus douglasii, in Vancouver, British Columbia, A Test of Interspecific Effects of Introduced Eastern Grey Squirrels, 10

Scorpion-grass, Blue, 320

Scoter, 232 Black, 233

Scoter Melanitta nigra, in Northumberland Strait, Interrupted by the Confederation Bridge, New Brunswick Prince Edward Island, An Observation of the Spring 2006 Migration of Black, 233

Scoter, Melanitta spp., Migrations Interrupted by Confederation Bridge: An Update, 232

Screech-Owl, Eastern, 289

Screech-Owl, Megascops asio, Population in an Apple-Producing Region of Southern Quebec, First Observations of an Eastern, 289

Sculpin, Deepwater, 480

Prickly, 425

Roughback, 364

Sculpin, Myoxocephalus thompsonii, in Northeastern Alberta, Seal, 466 A New Record of Deepwater, 480

Harbour, 62

Ringed, 464

Seburn, D., Review by, 248

Sedge, Awl-fruited, 22

Few-seeded, 22

Foothill, 160

Green-sheathed, 160

Lyngbei, 213

Sedum acre, 356

spathifolium, 154

Selatosomus pulcher, 19

Seminatrix pygaea, 477

Sericomyia chrysotoxoides, 17

Serrasalmus sp., 421

Shad, American, 422

Sheep, Bighorn, 314

Dall, 205

Shepherdia canadensis, 172 
Shiner, Redside, 421

Spottail, 422

Shoveler, Northern, 5

Shrew, Dusky, 332

Masked, 50,332

Northern Short-tailed, 436

Pygmy, 332

Short-tailed, 294

Shrimp, Spot, 364

Silene antirhina, 356

Simard, P., J.L. Lawlor, and S. Gowans. Temporal Variability of Cetaceans near Halifax, Nova Scotia, 93

Sisyrinchium arenicola, 359

fuscatum, 357

Sitta sp., 290

canadensis, 303

carolinensis, 303

Skeleton-weed, 336

Skimmer, Four-spotted, 417

Twelve-spotted, 417

Widow, 417

Skunk, Santa Cruz Island Spotted, 210 Striped, 229,342,452

Skunks, Mephitis mephitis, on Duck Nests in Saskatchewan, Summer Movements and Impact of Individual Striped, 342

Slough, B.G., 39,331

Slough, B.G. and R.L. Mennell. Diversity and Range of Amphibians of the Yukon Territory, 87

Snake, Black Swamp, 477

Maritime Garter, 477

Snake, Thamnophis sirtalis pallidulus, on Cape Breton Island, Nova Scotia, Bald Eagle, Haliaeetus leucocephalus, Preying on Maritime Garter, 477

Snow, D.P., 50

Solidago spp., 226

bicolor, 356

canadensis, 356

graminifolia, 356

juncea, 356

nemoralis, 356

puberula, 356

Somateria mollissima, 233,236

Somateria mollissima, Duckling, Osprey, Pandion haliaetus, Depredates Common Eider, 236

Somatochlora elongata, 415 kennedyi, 417

Soopolallie, 172

Sorex sp., 294

cinereus, 50,332

cinereus acadicus, 51

hoyi, 332

monticolus, 332

Soybean, 226

Sparganium angustifolium, 240

Sparrow, House, 43,264

Le Conte's, 22

Sparrow, Ammospiza leconteii, from Northeastern Ontario, with Some Notes on Nesting Behaviour, New Nesting Records of the Le Conte's, 22

Sparrows, Passer domesticus, in Canada's Maritime Provinces, Recent Declines of House, 43

Spartina, 414

alterniflora, 414

patens, 414 pectinata, 356,416

Speedwell, American, 158

Spring, 320

Spermophilus spp., 31

Sphaerophoria novaeangliae, 17

Sphagnum sp., 172,440

Sphegina brachygaster, 17

campanulata, 17

flavomaculata, 17

keeniana, 17

Sphyraena barracuda, 241

borealis, 241

guachancho, 241

Sphyraena barracuda from Canada, First Record of the Great Barracuda, 241

Sphyrapicus varius, 303

Spilogale gracilis amphiala, 210

Spiraea alba, 356

Spiranthes lacera, 356

Spray, Ocean, 158

Spreadwing, Elegant, 417

Lyre-tipped, 417

Northern, 417

Slender, 417

Spotted, 417

Sweetflag, 417

Spruce, 20,110,236,431

Black, 22,100,240,299,314,331,433

Englemann, 100

Norway, 299

Red, 299,440

Sitka, 11

White, 100,299,314,331

Spruce-fir, 331

Spurge, Leafy, 337

Squirrel, 295

Douglas's, 10

Eastern Grey, 10

European Red, 10

Gray, 264

Ground, 31

Red, 10,51,57

Squirrels, Sciurus carolinensis, on Douglas's Squirrels, Tamiasciurus douglasii, in Vancouver, British Columbia, A Test of Interspecific Effects of Introduced Eastern Grey, 10

Squirrels, Tamiasciurus douglasii, in Vancouver, British Columbia, A Test of Interspecific Effects of Introduced Eastern Grey Squirrels, Sciurus carolinensis, on Douglas's, 10

Star, Long-rayed, 364

Sunflower, 364

Starling, 264 European, 109,290

Steinhilber, M. and D.A. Neely. A New Record of Deepwater Sculpin, Myoxocephalus thompsonii, in Northeastern Alberta, 480

Stellaria graminea, 356 pallida, 320

Stickleback, 421

Brook, 422

Threespine, 240,422

Stonecrop, Lance-leaved, 154

Strauss, E.G., 263,478

Strawberry, 360 
Streever, B., 323

Strix varia, 290,303

Sturgeon, Lake, 71

White, 422

Sturgeon, Acipenser fulvescens, Movements in Rainy Lake, Minnesota and Ontario, Lake, 71

Sturnus vulgaris, 109,264,290

Stylasterias forriei, 364

Sucker, Longnose, 422,481

White, 422

Sunflower, Prairie, 336

Surnia ulula, 433

Surnia ulula, near Timmins and Iroquois Falls, Northeastern Ontario, in 2001, Observations on the Nesting of the Northern Hawk Owl, 433

Swallow, Tree, 302

Swan, Trumpeter, 220

Sweet-clover, 337

Swordtail, 422

Sylvilagus spp., 165 floridanus, 475 nuttallii, 165

Sympetrum sp., 416 costiferum, 415 danae, 413

internum, 415

obtrusum, 415

semicinctum, 417

vicinum, 415

Symphyotrichum laterifloum, 356

Synhalonia, 180

Syrphus rectus, 17

Szumylo, D.-L.M., 263

Tachycineta bicolor, 303

Tamarack, 314

Tamias striatus, 264,295 striatus lysteri, 51

Tamiasciurius sp., 295 douglasii, 10 hudsonicus, 10,57 hudsonicus ungavensis, 51

Tamiasciurus douglasii, in Vancouver, British Columbia, A Test of Interspecific Effects of Introduced Eastern Grey Squirrels, Sciurus carolinensis, on Douglas's Squirrels, 10

Tanichthys albonubes, 424

Taraxacum officinale, 320,358

Taricha granulosa, 90

Taxidea taxus, 31,209,342

Tea-berry, Western, 171

Tea, Labrador, 172

Tegler, B., 496

Tegler, B., Review by, 252

Temnostoma balyras, 17

Tench, 422

Thamnophis sirtalis pallidulus, 477

Thamnophis sirtalis pallidulus, on Cape Breton Island, Nova Scotia, Bald Eagle, Haliaeetus leucocephalus, Preying on Maritime Garter Snake, 477

Thiel, R.P. Conditions for Sexual Interactions Between Wild Grey Wolves, Canis lupus, and Coyotes, Canis latrans, 27

Thimbleberry, 171

Thrasher, Brown, 294
Thuja occidentalis, 300 plicata, $11,170,348$

Thymallus arcticus, 422

Tilapia sp., 422

Tilapia, 422

Tinca tinca, 422

Toad, American, 240 Western, 87

Toadflax, Dalmation, 176

Tokaryk, T., Reviews by, 127,485

Tonella tenella, 137,179

Tonella, Small-flowered, 137,179

Tonella tenella, in Canada, Conservation Evaluation of the Small-flowered Tonella, 179

Tonella, Tonella tenella, in Canada, Conservation Evaluation of the Small-flowered, 179

Torilis japonica, 180

Toxomerus geminatus, 17

Toxostoma rufum, 294

Tramea spp., 418

Trefoil, Bird's-foot, 157 Bog Bird's-foot, 153,157 Seaside Bird's-foot, 153,157

Trefoil, Lotus formosissimus, in Canada, Conservation Evaluation of the Seaside Birds-foot, 153

Trefoil, Lotus pinnatus, in Canada, Conservation Evaluation of the Bog Bird's-foot, 157

Tremblay, E., 438

Trientalis borealis, 356

Trifolium arvense, 357 procumbens, 357

Triglochin maritimum, 214

Tringa flavipes, 5

Triteleia hyacinthina, 158

Triteleia, White, 158

Troglodytes troglodytes, 303

Trout, Brook, 422

Brown, 422

California Golden, 422

Lake, 481

Rainbow, 90,199,421

Sea, 199

Troutperch, 422

Tsuga canadensis, 290 heterophylla, 11,170,348

Turdus migratorius, 294

Tursiops truncatus, 97

Twayblade, Heart-leaved, 170

Twinflower, 172

Typha latifolia, 214

Umbra limi, 238

Umbra limi, dans le nord-est du Québec, Extension de l'aire de distribution de l'Umbre de vase, 238

Urocyon littoralis, 210

Urosalpinx cinera, 106

Ursus americanus, 57,68,110,209,314 americanus hamiltoni, 51 arctos, 57,314

Ursus americanus, Retrieval of an Elk, Cervus elaphus, Carcass from a Small Lake in Riding Mountain National Park, Manitoba, Probable Black Bear, 110

Ursus americanus L., Long-range Homing by an Adult Female Black Bear, 57 
Vaccinium alaskense, 172

angustifolium, 357

angustifolium $\mathrm{X}$ corymbosum, 357

myrtilloides, 357

ovatum, 348

parvifolium, 11,172

scoparium, 172

Vander Kloet, S.P., 351

Varden, Dolly, 422

Vasseur, L., 438

Velvet-grass, Common, 154

Vernalgrass, Sweet, 148,154,160

Veronica beccabunga ssp. americana, 158 officinalis, 357 verna, 320

Vetch, Common, 180

Viburnum nudum var. cassinoides, 357

Vicia sp., 157

cracca, 357

sativa, 180

tetrasperma, 357

Viola sagittata var. ovata f. glabrata, 360 sagittata var. ovata, 357

Violette, V., 236

Vole, Eastern Heather, 332

Meadow, 50,264,294,332,436

Northern Red-backed, 331

Southern Red-backed, 50,331

Vole, Clethrionomys gapperi, in Newfoundland: Implications for the Endangered Newfoundland Marten, Martes americana atrata, First Record of the Southern RedBacked, 50

Vole, Myodes gapperi, in the Yukon, First Records of the Southern Red-backed, 331

Volucella bombylans, 17

Vors, L.S. and P.L. Wilson. A New Record Size Wolf, Canis lupus, Group for Ontario, 367

Vulpes fulva, 468

macrotis, 52

macrotis mutica, 210

velox, 31

vulpes, 36,68,263,342

vulpes deletrix, 51

Vulpes velox, in Southeastern Colorado, Multiple Scale Den Site Selection by Swift Foxes, 31

Walley, W.J. Probable Black Bear, Ursus americanus, Retrieval of an Elk, Cervus elaphus, Carcass from a Small Lake in Riding Mountain National Park, Manitoba, 110

Walley, W.J., Ring-billed Gull, Larus delawerensis, Food Piracy on Diving Ducks, 109

Walleye, 421

Walton, L.R., 342

Wasp, 295

Waxwing, Cedar, 294

Way, J.G. and D.L. Eatough. Use of "Micro"-Corridors by Eastern Coyotes, Canis latrans, in a Heavily Urbanized Area: Implications for Ecosystem Management, 474

Way, J.G., D.-L.M. Szumylo, and E.G. Strauss. An Ethogram Developed on Captive Eastern Coyotes Canis latrans, 263

Way, J.G., S.M. Cifuni, D.L. Eatough, and E.G. Strauss. Rat Poison Kills a Pack of Eastern Coyotes, Canis latrans, in an Urban Area, 478
Weasel, Short-tailed, 51,62

Weatherloach, 424

Wery, P., 289

Whale, Blue, 95

Bowhead, 462

Fin, 95

Humpback, 95

Killer, 457

Long-finned Pilot, 97

Minke, 95

North Atlantic Right, 95

Pilot, 97

Pygmy Sperm, 97

Sei, 97

Whale, Orcinus orca, Attacks in the Eastern Canadian Arctic, Reactions of Narwhals, Monodon monoceros, to Killer, 457

Wheat, 343

Wheat-grass, Bluebunch, 176

Crested, 337

Northern, 336

Slender, 184

Whiteface, Frosted, 417

Whitefish, Lake, 422,480

Whitetail, Common, 417

Whitlaw, H.A., 205

Whitlow-grass, Spring, 320

Wildlife Afield, 132,502

Williams, R., 199

Willis, D.W., 71

Willow, 158,314

Sandbar, 336

Wilson, P.L., 367

Windgrass, Interrupted, 320

Wissink, R., 438

Wolf, 27,57,67,263,313,367,466,471,476,478

Arctic, 466,471

Eastern, 27

Eastern Timber, 51

Gray [Grey], 27,61,111,205,367

Newfoundland, 50

Red, 27

Timber, 27

Wolf, Canis lupus, Group for Ontario, A New Record Size, 367

Wolverine, 11

Wolves, Canis lupus arctos: Influence of Order on Posture, Double Marking in Arctic, 471

Wolves, Canis lupus, and Coyotes, Canis latrans, Conditions for Sexual Interactions Between Wild Grey, 27

Wolves, Canis lupus arctos, in Summer, Urine-marking and Ground-scratching by Free-ranging, 466

Wolves, Canis lupus, in Southcentral Alaska, Physical Characteristics, Hematology, and Serum Chemistry of Freeranging Gray, 205

Wolves, Canis lupus, of Coastal British Columbia, Early Ontogenetic Diet in Gray, 61

Wolves, Canis lupus, on Caribou, Rangifer tarandus, Winter Ranges in Westcentral Alberta, Pack Size of, 313

Woodley, S.J., G. Johnson, B. Freedman, and D.A. Kirk. Effects of Timber Harvesting and Plantation Development on Cavity-nesting Birds in New Brunswick, 298

Woodpecker, Black-backed, 302 Downy, 302 
Hairy, 294,302

Pileated, 299,302

Woolly-heads, Dwarf, 163

Woolly-heads, Psilocarphus brevissimus var. brevissimus, in Canada, Conservation Evaluation of the Pacific Population of Dwarf, 163

Wren, Winter, 302

Xiphophorus helleri, 422

Xylota, 20

confusa, 17

quadrimaculata, 17

\section{Index to Book Reviews}

\section{Botany}

Burger, W.C. Flowers How They Changed the World, 249

Carder, A. Giant Trees of Western America and the World, 120

Rover, F. and R. Dickinson. Plants of Alberta, 386

Scotter, G. and H. Flygare. Wildflowers of the Rocky Mountains, 386

Viereck, L. and E.L. Little. Alaska Trees and Shrubs. Second Edition, 491

Environment

Dow, K. and T.E. Downing. The Atlas of Climate Change: Mapping the World's Greatest Challenge, 493

Fish and Wildlife Historical Society. Fish, Fur and Feathers: Fish and Wildlife Conservation in Alberta, 1905-2005, 124

Gore, A. An Inconvenient Truth: The Planetary Emergency of Global Warming and What We Can Do About It, 494

Kruse, G.H., V.F. Gallucci, D.E. Hay, R.I. Perry, R.M. Peterman, T.C. Shirley, P.D. Spencer, B. Wilson, and, D. Woodby. Fisheries Assessment and Management in Data-Limited Situations, 121

McGavin, G.C. Endangered: Wildlife on the Brink of Extinction, 252

Mundy, P. The Gulf of Alaska: Biology and Oceanography, 125

Redcliff, D. Lapland A Natural Historym 123

Saab, V.A. and H.D.W. Powell. Fire and Avian Ecology in North America Studies in Avian Biology Number 30,121

Warren, A. and T. Allan. Guide to Deserts, 251

\section{Miscellaneous}

Burt, W. Marshes: The Disappearing Edens, 389

Callan, K. A Paddler's Guide to Quetico and Beyond, 496

Czajkowski, C. Wildfire in the Wilderness, 498

Elder, J. Pilgrimage to Vallombrosa: From Vermont to Italy in the Footsteps of George Perkins Marsh, 387

Hudson, R. A Field Guide to Gold, Gemstones \& Mineral Sites of British Columbia, Sites Within a Day's Drive of Vancouver, 128

Hudson, R. A Field Guide to Gold, Gemstones \& Mineral Sites of British Columbia, Sites Within a Day's Drive of Vancouver, 253

Koeppel, D. To See Every Bird on Earth, 498

Mabey, R. Gilbert White: A Biography of the Author of The Natural History of Selborne, 496

Mayor, A. Fossil Legends of the First Americans, 127

Milne, P. Where to Watch Birds World Cities, 388

Mock. D.W. More than Kin and Less than Kind: The Evolution of Family Conflict, 497
Yellow-cedar, 172

Yellowlegs, Lesser, 5

Yukon, First Records of the Southern Red-backed Vole, Myodes gapperi, in the, 331

Yukon Territory, Diversity and Range of Amphibians of the, 87

Yukon Territory, First Records of the Northern Long-eared Bat, Myotis septentrionalis, in the, 39

Zannichellia palustris, 416

Zapus hudsonius, 332

Zea mays, 226

Zenaida macroura, 294

Quammen, D. The Reluctant Mr. Darwin: An Intimate Portrait of Charles Darwin and the Making of His Theory of Evolution, 252

Shtilmark, F. History of the Russian Zapovedniks 1985-1995, 254

Van der Flier-Keller, E. A Field Guide to the Identification of Pebbles, 255

Zoology

Acorn, J. Deep Alberta. Fossil Facts and Dinosaur Digs, 485

Acorn, J. Ladybugs of Alberta: Finding the Spots and Connecting the Dots, 486

Adler, P., D. Currie, and D.M. Wood. The Black Flies (Simuliidae) of North America, 245

Arlott, N. Birds of Europe, Russia, China and Japan: Passerines, Tyrant Flycatchers to Buntings, 484

Beadle, D. and J. Rising. Tanagers, Cardinals, and Finches of the United States and Canada, 119

Bildstein, K.L. Migrating Raptors of the World: Their Ecology and Conservation, 382

Bonin, F., B. Devaux and A. Dupre. Turtles of the World, 248

Chu, M. Songbird Journeys: Four Seasons in the Lives of Migratory Birds, 385

Clements, J.F. The Clements Checklist of Birds of the World 6th Edition, 483

de Asua, M. and R. French. A New World of Animals: Early Modern Europeans on the Creatures of Iberian America, 487

del Hoyo, J., A. Elliott and D.A. Christie. Handbook of the Birds of the World Volume II Old World Flycatchers to Old World Warbler, 381

Eisner, T., M. Eisner, M. Siegler. Secret Weapons Defenses of Insects, Spiders, Scorpions, and Other Many-Legged Creatures, 118

Elbroch, M. Animal Skulls, A Guide to North American Species, 243

Elphick, J. Atlas of Bird Migration: Tracing the Great Journeys of the World's Birds, 380

Evans, H.E. and K.M. O'Neill. The Sand Wasps: Natural History and Behavior, 489

Firouz, E. The Complete Fauna of Iran, 114

Forsyth, R.G. Land Snails of British Columbia, 383

Gibson, M. Gibson's Guide to Bird Watching \& Conservation, 485

Hoogland, J. Conservation of the Black-tailed Prairie Dog: Saving North America's Western Grasslands, 382

Jackson, D.C. Tracks, 490

Latta, S., C. Rimmer, A. Keith, J. Wiley, H. Raffaele, K. McFarland, E. Fernandez. Birds of the Dominican Republic and Haiti, 379 
Lynch, W. Penguins of the World, 488

Marshall, S.A. Insects: Their Natural History and Diversity: With a photographic guide to insects of eastern North America, 117

Marshall, S.A. Insects: Their Natural History and Diversity: With a Photographic Guide to Insects of Eastern North America, 246

Maybank, B. The Birding Sites of Nova Scotia, 114

Morrison, M.L. The Northern Goshawk: A technical Assessment of its Status, Ecology and management, 247

Moss, S. The Birder's Companion, 379

Nagorsen, D.W. Rodents and Lagomorphs of British Columbia, 118

Nelson, J.S. Fishes of the World, 116

O'Connor, M. Why Don't Woodpeckers Get Headaches? And Other Bird Questions You Know You Want To Ask, 490
Panov, E.N. Wheatears of Palaearctic: Ecology, Behaviour and Evolution of the Genus Oenanthe, 119

Proctor, N. and P. Lynch. A Field Guide to North Atlantic Wildlife, 244

Sale, R. A Complete Guide to Arctic Wildlife, 244

Stevenson, T. and J. Fanshawe. The Birds of East Africa: Kenya, Tanzania, Uganda, Rwanda, Burundi, 113

Theberge, J.B. and M.T. Theberge. The Wolves of Algonquin Park: A 12-year Ecological Study, 249

Wheeler, B.K. Raptors of Western North America and Raptors of Eastern North America, 489

Young Naturalists

de Vries, M. Tale of a Great White Fish, 499 


\section{Advice for Contributors to The Canadian Field-Naturalist}

\section{Content}

The Canadian Field-Naturalist is a medium for the publication of scientific papers by amateur and professional naturalists or field biologists reporting observations and results of investigations in any field of natural history provided that they are original, significant, and relevant to Canada. All readers and other potential contributors are invited to submit for consideration their manuscripts meeting these criteria. The journal also publishes natural history news and comment items if judged by the Editor to be of interest to readers and subscribers, and book reviews. Please correspond with the Book Review Editor concerning suitability of manuscripts for this section. For further information consult: A Publication Policy for the Ottawa Field-Naturalists' Club, 1983. The Canadian Field-Naturalist 97(2): 231-234. Potential contributors who are neither members of The Ottawa Field-Naturalists' Club nor subscribers to The Canadian Field-Naturalist are encouraged to support the journal by becoming either members or subscribers.

\section{Manuscripts}

Please submit by post to the Editor, in either English or French, three complete manuscripts written in the journal style. Manuscripts may also be submitted (one copy) by email. The research reported should be original. It is recommended that authors ask qualified persons to appraise the paper before it is submitted. All authors should have read and approved it. Institutional or contract approval for the publication of the data must have been obtained by the authors. Also authors are expected to have complied with all pertinent legislation regarding the study, disturbance, or collection of animals, plants or minerals. The place where voucher specimens have been deposited, and their catalogue numbers, should be given. Latitude and longitude should be included for all individual localities where collections or observations have been made.

Manuscripts should be printed on standard-size paper, doublespaced throughout, generous margins to allow for copy marking, and each page numbered. For Articles and Notes provide a bibliographic (citation) strip, an abstract, and a list of key words. Generally, words should not be abbreviated but use SI symbols for units of measure. The names of authors of scientific names may be omitted except in taxonomic manuscripts or other papers involving nomenclatural problems. "Standard" common names (with initial letters capitalized) should be used at least once for all species of higher animals and plants; all should also be identified by scientific name.

The names of journals in the Literature Cited should be written out in full. Unpublished reports and web documents should not be cited here but placed in a separate Documents Cited section. List the captions for figures numbered in arabic numerals and typed together on a separate page. Present the tables each titled, numbered consecutively in arabic numerals, and placed on a separate page. Mark in the margin of the text the places for the figures and tables.
Check recent issues (particularly Literature Cited) for journal format. Either "Canadian" or "American" spellings are acceptable in English but should be consistent within one manuscript. The Oxford English Dictionary, Webster's New International Dictionary and le Grand Larousse Encyclopédique are the authorities for spelling.

\section{Illustrations}

Photographs should have a glossy finish and show sharp contrasts. Electronic versions should be high resolution. Prepare line drawings with India ink on good quality paper and letter (don't type) descriptive matter. Photographic reproduction of line drawings should be no larger than a standard page. Write author's name, title of paper, and figure number on the lower left corner or on the back of each illustration.

\section{Reviewing Policy}

Manuscripts submitted to The Canadian Field-Naturalist are normally sent for evaluation to an Associate Editor (who reviews it or asks another qualified person to do so), and at least one other reviewer, who is a specialist in the field, chosen by the Editor. Authors are encouraged to suggest names of suitable referees. Reviewers are asked to give a general appraisal of the manuscript followed by specific comments and constructive recommendations. Almost all manuscripts accepted for publication have undergone revision-sometimes extensive revision and reappraisal. The Editor makes the final decision on whether a manuscript is acceptable for publication, and in so doing aims to maintain the scientific quality, content, overall high standards and consistency of style, of the joumal.

\section{Special Charges - Please take note}

Authors must share in the cost of publication by paying $\$ 90$ for each page, plus $\$ 30$ for each illustration (any size up to a full page), and up to $\$ 90$ per page for tables (depending on size). Authors may also be charged for their changes in proofs. Reproduction of color photos is extremely expensive; price quotations may be obtained from the Editor.

Limited joumal funds are available to help offset publication charges to authors without grants or institutional support. Requests for financial assistance should be made to the Editor when the manuscript is submitted.

\section{Reprints}

An order form for the purchase of reprints or pdf will accompany the galley proofs sent to the authors. Invoices for publication costs will be sent when the submission is published.
FRANCIS R. COOK, Editor RR 3 North Augusta, Ontario K0G IR0 Canada 